\title{
Uso de dilemas morales en la formación de la conciencia moral
}

\author{
Omar León", Freddy Patiño**, Mauricio Buitrago ${ }^{* * *}$, \\ Johanna Arias ${ }^{* * * *}$, Blanca Rosmira Meza $a^{* * * *}$
}

Recibido: 4 de noviembre de 2009 Revisado: 11 de diciembre de 2009 Aprobado: 6 de febrero de 2010

\section{RESUMEN}

Este artículo presenta los resultados de la investigación Efecto de una estrategia pedagógica basada en el uso de dilemas morales en el desarrollo de la conciencia moral de estudiantes de secundaria, adelantada en el marco de la Maestría en Educación de la Universidad Santo Tomás, y que hace parte del macroproyecto de investigación Efecto de una estrategia pedagógica en el desarrollo de la conciencia moral en un grupo de estudiantes universitarios. El proyecto, que asume como presupuestos teóricos los planteamientos de Laurence Kolhberg y su definición de seis estadios del desarrollo moral agrupados en tres niveles (preconvencional, convencional y posconvencional), se ubica en el paradigma positivista y asume desde el método cuantitativo un diseño cuasiexperimental (con un grupo experimental y un grupo de control no equivalente). La herramienta de medición, utilizada al iniciar y al concluir el proceso de intervención, es el Defining Issues Test, DIT, diseñado por James Rest. El proyecto permitió constatar que, aplicada la estrategia pedagógica, a) el grupo de control concentra su ubicación en el nivel convencional del desarrollo moral, mientras que b) el grupo experimental evidencia una tendencia de crecimiento de los estadios inferiores a los superiores, específicamente un incremento en el porcentaje de estudiantes que se ubican en el estadio posconvencional.

\section{Palabras clave}

Desarrollo moral, estrategia pedagógica, conciencia moral, dilema moral, estadio moral

* Omar Asdrúbal León Carreño: Licenciado en Filosofía y Letras de la Universidad Santo Tomás y Magíster en Educación de la misma institución. Docente investigador de la Universidad Santo Tomás. Dirección electrónica: omar.leon.c@ gmail.com.

** Freddy Patiño Montero: Licenciado en Filosofia de la Universidad Santo Tomás y Magister en Educación de la misma institución, Docente de la Fundación Universitaria Los Libertadores y de la Fundación Universitaria Panamericana. Dirección electrónica: freddypam@ hotmail.com.

*** Mauricio Esteban Buitrago Ropero: Licenciado en Electrónica de la Universidad Pedagógica Nacional, Especialista en Informática Educativa de la Universidad Central de Colombia y y Magíster en Educación de la Universidad Santo Tomás. Docente de la Universidad Pedagógica Nacional y de la Fundación Universitaria del Área Andina. Dirección electrónica: mauyricioest@ yahoo.com.

****Johanna Andrea Arias Gutérrez: Licenciada en Educación para la Infancia de la Universidad Distrital Francisco José de Caldas y Magister en Educación de la Universidad Santo Tomás. Docente del Colegio Distrital Saludcoop Sur. Dirección electrónica: jaariasgu05@ yahoo.es.

***** Blanca Rosmira Meza Pérez: Licenciada en Básica Primaria de la Universidad Antonio Naniño y Magíster en Educación de la Universidad Santo Tomás. Docente del Colegio Distrital Saludcoop Sur. Dirección electrónica: rosmiraa@ hotmail.com. 


\title{
Use of moral dilemmas in the development of the moral consciousness
}

\author{
Omar León, Freddy Patiño, Mauricio Buitrago, Johanna Arias, Blanca Rosmira Meza
}

\begin{abstract}
This article reports the results of the research project titled "Effect of a pedagogic strategy based on the use of moral dilemmas in the development of the moral consciousness of high school students". This study was developed in the Master's in Education program at the Universidad Santo Tomás (Colombia) as part of the macroproject "Effect of a pedagogic strategy based on the use of moral dilemmas in the development of the moral consciousness in a group of university students". The project relies on the theoretical foundations posed by Laurence Kohlberg and his definitions concerning the six stages of moral development, grouped in three levels: pre-conventional, conventional and post-conventional. This study is framed within the positivist paradigm and follows a quantitative method with a quasi-experimental design. The instrument used to collect and analyze data is the Defining Issues Test (DIT) designed by James Rest. Results showed that a) the control group is located in the conventional stage of moral development, b) the experimental group, shows an increasing tendency to move from inferior to superior stages, especially an increase in the average number of students who are located in the post-conventional stage.
\end{abstract}

\section{Key words}

Moral development, pedagogical strategy, moral conscience, moral dilemma, moral stage. 


\section{INTRODUCCIÓN}

En la actualidad ha venido cobrando mayor relevancia la formación de seres humanos capaces de establecer relaciones armónicas con sus pares y con el medio en el que se desenvuelven. Por esta razón, toman fuerza los temas relacionados, por ejemplo, con la construcción de una mejor comprensión de lo que significa ser ciudadanos y, en esta medida, de las implicaciones que tienen las acciones del ser humano, puesto que cada una de estas trae consigo consecuencias para sí mismo y para los demás. Ello reclama afrontar de nuevo el debate acerca de la moralidad de los actos y de la conciencia moral como aspecto fundamental en el momento de tomar decisiones, de dar razón de las motivaciones que las sustentan o de asumir responsablemente las consecuencias de las mismas.

Es en este horizonte en el que se ubica el presente trabajo, que asume como punto de partida las investigaciones Formación de la conciencia moral en la Educación Superior, adelantada por Suárez y Meza (2005), a partir de cuyos resultados se llevó a cabo en 2008 el proyecto Efecto de una estrategia pedagógica en el desarrollo de la conciencia moral en un grupo de estudiantes universitarios, por parte de los mismos investigadores, el cual se cuestionaba sobre la posibilidad de formar la conciencia moral y ubicaba dicho cuestionamiento en el ámbito específico de los estudios universitarios formales; la respuesta a la que llegaron con tal trabajo fue positiva y señalaron en el curso de la misma dos posibilidades para el desarrollo de la conciencia moral: por un lado, una vía basada en la no intervención, según la cual la sola inmersión e interacción del individuo en un ambiente cultural determinado basta para que este se desarrolle moralmente, pues la cultura le aporta suficientes elementos para ir escalando en cada una de las etapas del desarrollo moral. No obstante, hacían evidente, en el marco de nuestra cultura, la necesidad de acudir a una vía alterna, la vía de la intervención directa e intencionada, recurriendo a espacios educativos concretos de nuestro contexto colombiano para promover el desarrollo moral de la persona.

Tal investigación constituye el punto de partida del macroproyecto investigativo del mismo nombre, enmarcado en la línea de investigación Formación, convivencia y ciudadanía, de la Maestría en Educación de la Universidad Santo Tomás, del cual forma parte este trabajo. Como parte del mismo, este proyecto asume como objetivo fundamental la determinación del efecto de una estrategia pedagógica basada en el uso de dilemas morales en estudiantes de secundaria. Los resultados del presente proyecto, unidos a los demás proyectos que de manera paralela se adelantan en esta fase, aportan al macroproyecto los datos y las experiencias de orden pedagógico necesarias para el análisis de la validez y pertinencia de la propuesta de formación de la conciencia moral de estudiantes de diferentes ambientes educativos en el contexto colombiano.

En esta línea se indagó acerca de otras investigaciones que abordaran el problema (Oser, 1998, 2005; Thoma y Rest, 1999), particularmente en la realidad latinoamericana (Pompa et al., 2001; Barba, 2001, 2002; De Bennato, 2009) y colombiana (Villegas, 1998; Bermúdez y Jaramillo, 2000; Botero Chica, 2005), y se abordaron los presupuestos teóricos que dan soporte a la pregunta misma y aportan los fundamentos desde los cuales se adelantan los respectivos análisis. 
Ya desde sus orígenes, el pensamiento humano ha abordado ampliamente el problema moral, y dentro de él la discusión respecto a la ley moral y la libertad de la voluntad. Del intelectualismo ético del mundo clásico a la ética de situación de nuestros días, podríamos decir que el hombre no ha hecho otra cosa que tratar de dar rostro y significado a sus acciones, unos negando la norma y otros la libertad, elementos ambos esenciales, sin los cuales perdería sentido el problema moral mismo. En virtud de ello se consideró pertinente, en primer lugar, delinear algunos conceptos básicos en torno a la moralidad, a las características de esta dimensión del ser humano, en orden a precisar, por ejemplo, qué queremos significar al hablar de conciencia moral o al decir que el ser humano es sujeto moral (Becattini, 1999; Musco, 2008), y de dónde emerge esa característica particular, así como el lugar que ocupa en la vida personal y social (Dawkins, 1993; Hauser, 2006; Huxley, 1893; De Waal, 2007; Kolakowski, 1993; Riechmann, 2009; Hogan, 1973, 1975). En segundo lugar se ahonda en las posibilidades de formación de la conciencia moral, refiriendo diversas teorías que se consideran pertinentes (González, 2003; Kohlberg, 1983; Lehalle et al., 2004; Rest y Deemer, 1986; Bandura, 1986), con particular énfasis en la teoría de Lawrence Kohlberg, para pasar a establecer algunos rasgos que pueden verse como determinantes en el desarrollo moral del adolescente, ámbito en el que se ubica la población en la que se concentra nuestro trabajo (Wenek, 1996; Hersh, et al., 1998; Gibbs y Schnell, 1985; Schlaefli, et al., 1985; Suárez y Meza, 2008).

La idea básica de la teoría de Kohlberg es que el desarrollo moral implica la evolución de las estructuras o esquemas considerarse mentales que se producen generalmente según una sucesión invariable de seis estadios, agrupados de a dos en tres grandes niveles de desarrollo. Los estadios siguen un orden jerárquico, lo que equivale a decir que el razonamiento en estadios superiores tiende a suplantar el esquema de justificación moral de los inferiores. Sin embargo, puede suceder que una persona aplique razonamientos de estadios superiores o inferiores a su estadio predominante. Kohlberg llega a la conclusión de que la mayoría de los adultos operan generalmente en el estadio 4 y que solo un 15\% alcanzan el 5, y 6\% el 6.

El análisis conceptual de esta sucesión de estadios evidencia claramente la coherencia del sistema propuesto. Es fácil en efecto establecer que cada estadio del desarrollo se basa necesariamente en el anterior. Así, por ejemplo, no se puede llegar a considerar la ley como relativa (estadio 5) si previamente no se construye una representación de su función social (estadio 4); de la misma manera, para apreciar el funcionamiento social en su conjunto (estadio 4) se ve necesario haber experimentado el funcionamiento de los grupos sociales a escala más reducida en los grupos cercanos (estadio 3).

Lo que la teoría sobre el tema del desarrollo moral y los instrumentos actuales de estudio sobre el mismo permiten, incluido el DIT de James Rest y la entrevista semiestructurada de Kohlberg, es tener un modelo de subjetividad cognitiva desde el cual comprender el complejo sistema moral del ser humano. Por tanto, como investigadores usamos este sistema de referencia conceptual y metodológico para acercarnos a los grupos tanto experimental como de control y para el desarrollo de las intervenciones. 


\section{Metodología}

Para satisfacer su objetivo, la investigación se propuso un análisis del fenómeno del desarrollo moral desde el paradigma positivista, por lo que asumió el método cuantitativo y un diseño cuasiexperimental, de allí que se utilice el Defining Issues Test, DIT, de James Rest, que tiene por objeto la recolección de datos que permitan caracterizar un grupo poblacional (en nuestro caso, uno de control y uno experimental) frente a lo que la teoría del desarrollo moral de Kohlberg propone en relación con los estadios del desarrollo moral de la persona (Vargas Guillén, 2004; Hernández, 1998; Suárez y Meza, 2004; Buendía, 1998). Con el uso de dicho instrumento se estableció en primer lugar el nivel de desarrollo de la conciencia moral en los estudiantes que formaron parte de la muestra, se puso luego en práctica la estrategia pedagógica basada en dilemas morales en el grupo experimental, y se procedió a aplicar nuevamente el test y a comparar el nivel de desarrollo de la conciencia moral del grupo de estudiantes que recibieron la estrategia pedagógica frente al grupo que no la recibió, en orden a establecer si se cumplía la hipótesis planteada, a saber, que "los estudiantes partícipes del uso de la estrategia pedagógica basada en la aplicación de dilemas morales muestran un mayor desarrollo de la conciencia moral comparado con aquellos que no participan de esta".

La población escogida para el desarrollo de esta investigación son estudiantes del Gimnasio Campestre San Francisco de Sales, institución confesional católica con una filosofía humanista orientada por la propuesta educativa salesiana, ubicado en el municipio de Cota, Cundinamarca, cuya mayoría de estudiantes proviene del noroccidente de la ciudad de Bogotá, de la que los separan unos 30 minutos por vía terrestre. Los estudiantes, que contaban al iniciar con una edad promedio de 14 años, cursaban grado noveno en el año 2008, en cuyo segundo semestre se dio inicio a las intervenciones realizadas en el marco del presente proyecto, y cursaban grado décimo durante el primer semestre de 2009, en el cual termina la fase de aplicación. La mayoría de estos estudiantes pertenecen a familias configuradas de manera tradicional, con imágenes paternas y maternas fortalecidas. El estrato socioeconómico promedio es 4, aunque algunos de ellos pertenecen a los estratos 5 y 6 .

El grado noveno lo conformaban dos cursos, cada uno con un promedio de 30 estudiantes. Dichos cursos, y por lo tanto los grupos de trabajo, eran de carácter mixto y homogéneos entre sí, y para determinar el grupo experimental y el de control se hizo la elección de manera aleatoria para no viciar el proyecto. De este total salió la muestra, constituida según los resultados arrojados por los análisis del pretest, de acuerdo con los criterios de validación propios del instrumento aplicado, el DIT de James Rest.

El grupo de control sólo fue objeto de la aplicación del pretest y del postest, mientras en el grupo experimental se llevó a cabo, entre estos dos momentos, la aplicación de la estrategia pedagógica, constituida por siete intervenciones, que se asumen aquí como variable independiente (VI) y que se realizaron en el transcurso de cerca de diez meses entre los años 2008 y 2009. En cada una de las intervenciones se propuso una situación dilémica, la cual se usó como base para el inicio de una discusión de carácter formativo frente al dilema moral que encierra cada propuesta de intervención. 
En ellas se indagó y promovió la argumentación moral frente a situaciones de difícil resolución por parte de los miembros del grupo experimental.

Atendiendo al diseño metodológico definido, se estableció nuevamente el nivel de desarrollo moral a partir de la aplicación del instrumento, el cual arrojó los datos necesarios para contrastar con los ofrecidos por el pretest, contrastación de la cual emergen los resultados que a continuación se presentan.

\section{ANálisis y RESUltados}

Los resultados se sintetizan en cinco núcleos de análisis planteados a partir de las relaciones que pueden establecerse entre los grupos y fases del proyecto.

\section{Análisis y comparación del nivel inicial de desarrollo moral de los grupos experimental y de control}

Se puede afirmar la similitud entre los dos grupos respecto a la forma de asumir y actuar frente a diferentes situaciones sociales con implicaciones morales, dada la cercanía en los valores obtenidos en cada uno de los tres niveles de desarrollo. Así, según puede apreciarse en la figura 1, la diferencia en el porcentaje de estudiantes que se ubican en el nivel preconvencional es de apenas $1,25 \%$, siendo levemente mayor en el grupo de control. Es en cambio levemente superior el porcentaje de alumnos del grupo experimental que se ubica en el nivel convencional, con una diferencia de $1,26 \%$. Por último, la diferencia resulta ínfima para el nivel posconvencional, con solo el $0,01 \%$.

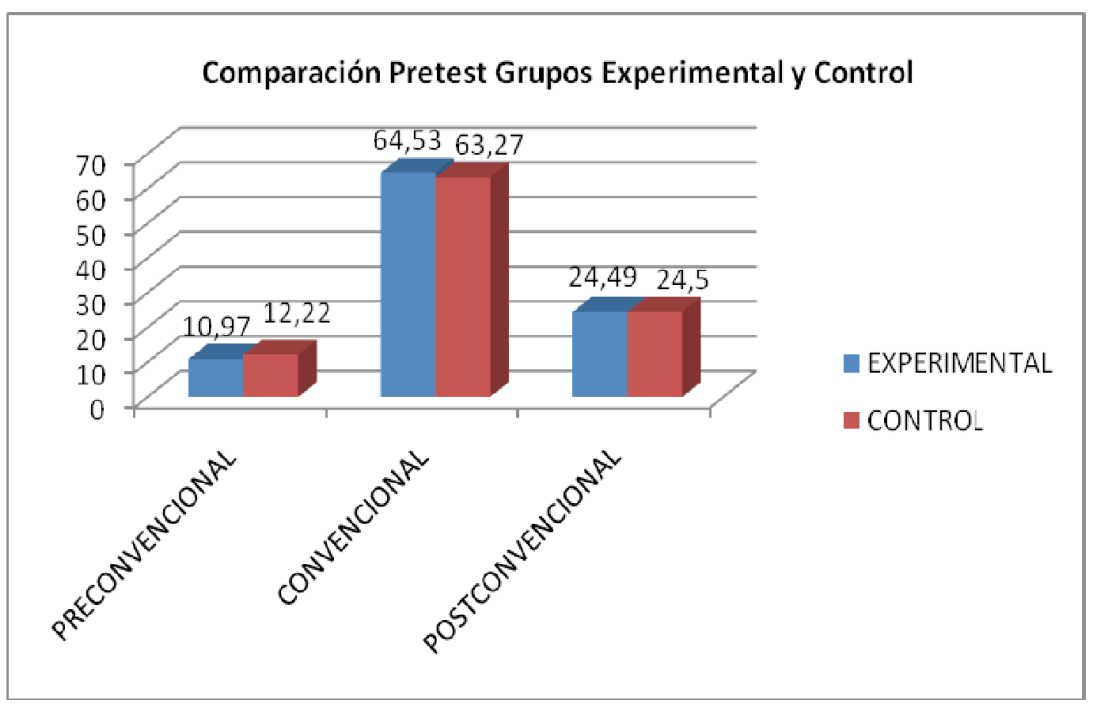

Figura 1. Comparación pretest grupos experimental y de control.

Puede afirmarse, en primer lugar, que ambos grupos muestran un nivel de desarrollo moral acorde con su edad en la perspectiva señalada por la teoría de Kohlberg, según la cual existe un vínculo entre la maduración biológica y el desarrollo moral que permite afirmar que es propio de los adolescentes el ubicarse en el nivel convencional. A su vez, 
tal similitud es oportuna en la perspectiva metodológica, que exige buscar la mayor homogeneidad posible entre los grupos experimental y de control, en orden a que los cambios producidos en el curso de la estrategia pedagógica le sean atribuibles a la misma y no a aquellas variables que escapan al control por parte del grupo de investigadores.

\section{Comparación del nivel posterior de desarrollo moral de los grupos experimental y de control}

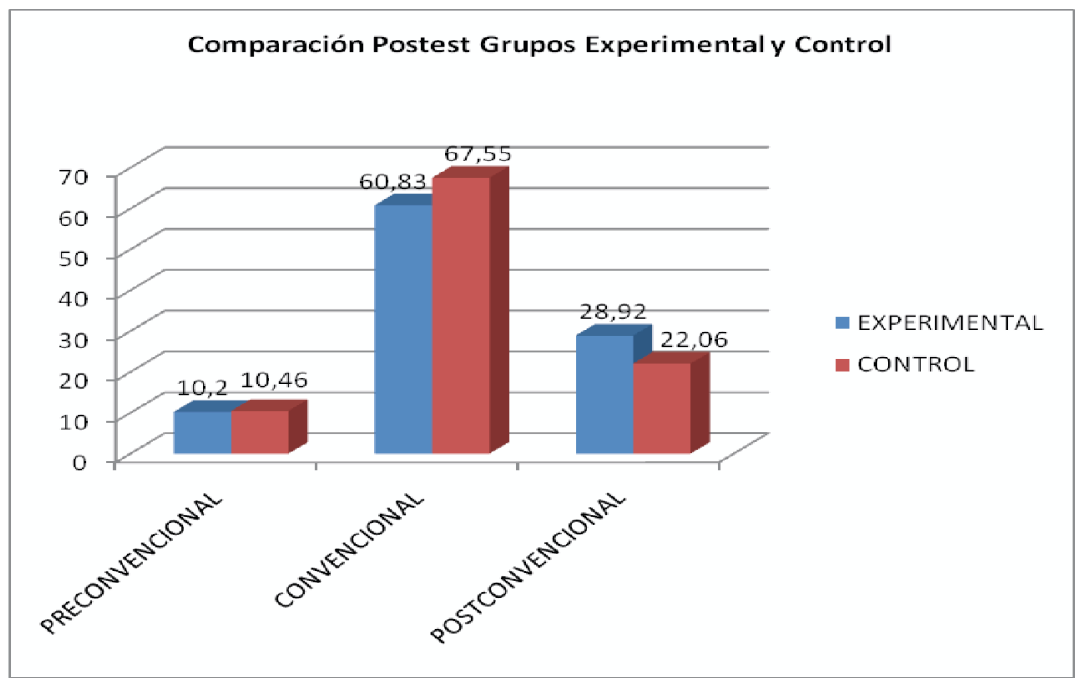

Figura 2. Comparación postest grupos experimental y control.

Transcurrido el periodo de tiempo en el cual se aplicó la estrategia pedagógica en el grupo experimental, la primera constatación que puede hacerse es la cercanía de los porcentajes de estudiantes que se ubican en el nivel preconvencional en ambos grupos, con una sutil diferencia de $0,26 \%$ del grupo experimental sobre el de control, según puede observarse en la figura 2, en oposición al momento inicial, en el que el grupo de control mostró un mayor porcentaje de alumnos en este nivel. No es en cambio sutil la diferencia que se da en los niveles restantes; en efecto, los puntajes del nivel convencional muestran una diferencia de $6,72 \%$ a favor del grupo de control sobre el experimental, que ratifica la tendencia de aquel a la permanencia general en el nivel convencional. En el nivel posconvencional la diferencia es también sustantiva y aún más marcada, alcanzando un $6,86 \%$, pero ahora del grupo experimental sobre el grupo de control, lo que evidencia la tendencia de crecimiento en los índices de desarrollo de la conciencia moral hacia el nivel posconvencional que se da en el grupo experimental.

La relevancia de estos datos se hace más clara al comparar los resultados de cada uno de los grupos en los dos momentos de aplicación del instrumento. 


\section{Análisis y comparación de los resultados de pretest y postest en el grupo de control}

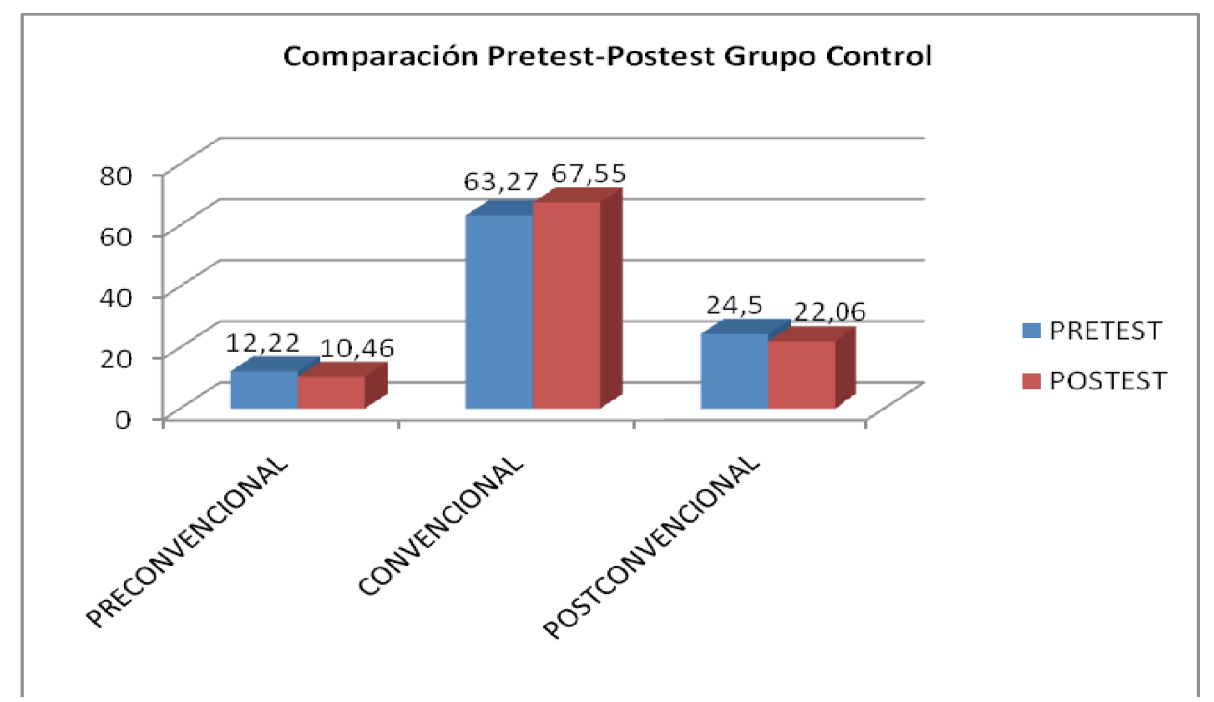

Figura 3. Comparación pretest-postest grupo control.

Al comparar, a través de la figura 3, los resultados obtenidos por el grupo de control tanto en el pretest como en el postest, se puede constatar que concentra su ubicación en el nivel convencional, que aumenta en un $4,28 \%$ al tiempo que decrece en un $1,76 \%$ en el nivel preconvencional y un $2,44 \%$ en el posconvencional. Simultáneamente, el análisis de los resultados arrojados por el instrumento respecto a las categorías $\mathrm{M}$ y $A^{\star}$, que tipifican el rango de distracción o rechazo ante el instrumento, permiten apreciar una disminución del puntaje $M$, que pasó de 134 puntos a 63, lo que equivale a un 52,98\%, y una reducción en el puntaje A, que pasó de 107 a 46, lo que indica una diferencia porcentual de $57 \%$.

De lo anterior puede afirmarse que el grupo, en relación con los resultados revelados por el pretest, se afianzó en el nivel convencional. Dada la expectativa de maduración cognitivo-evolutiva de los estudiantes en virtud de los procesos atinentes a su edad y educación, podría esperarse que sus niveles convencional y posconvencional aumentaran, sin embargo se presenta más bien una reducción de las respuestas que señalan el nivel posconvencional. Tal decrecimiento podría explicarse en virtud de la disminución de los puntajes $\mathrm{M}$ y A, que señalan mayor nivel de comprensión del instrumento o un ejercicio de respuesta más atento $\mathrm{y}$ dispuesto; ello significaría que la respuesta dada en el pretest se razonó menos y afectó el resultado final mostrando un valor superior, mientras que la mayor atención prestada en el momento de responder al postest reveló de manera más fehaciente la ubicación real de los estudiantes en los niveles respectivos, lo que permite identificar el convencional como aquel en que se ubican la mayoría de ellos.

Utilizamos el verbo en potencial ("significaría") en virtud de que es una de las conclu- 
siones posibles, dada sí a partir del análisis de los elementos constitutivos del DIT, pero que escapa a todas las variables que pudieron influir en el resultado, pues sin darse ningún tipo de intervención directa sobre el grupo de control cualquier afirmación que vaya más allá de lo que el mismo test revela se ubica en el ámbito de la especulación.

\section{Comparación del pretest y del postest del grupo experimental}

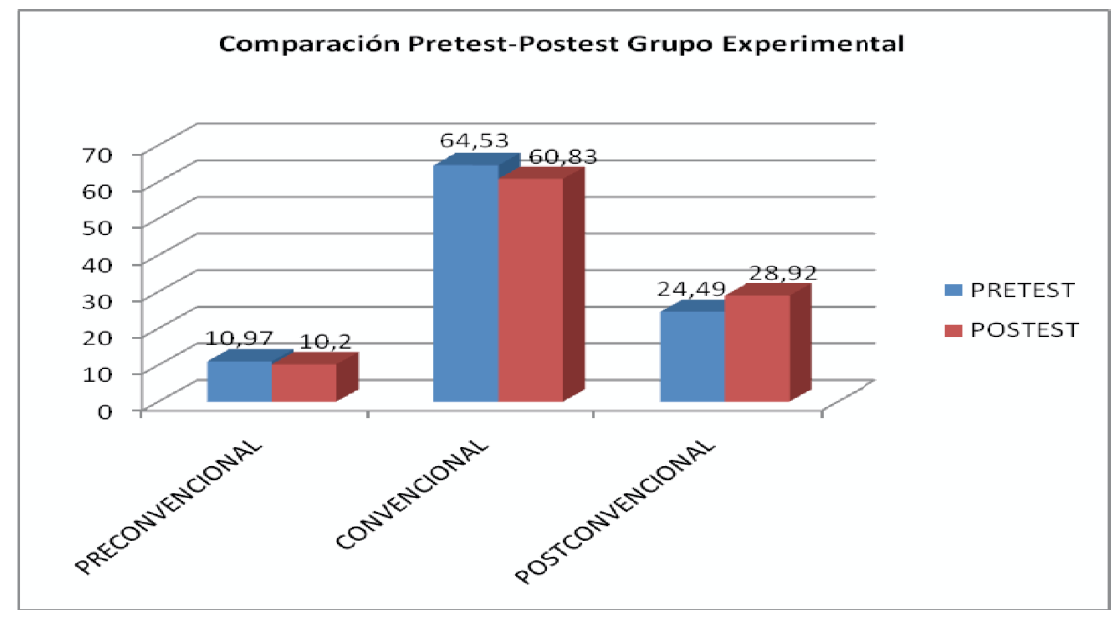

Figura 4. Comparación pretest-postest grupo experimental.

Los resultados que se presentan en este apartado tienen particular significación en el marco de la presente investigación, puesto que permiten ver el comportamiento que tuvo el grupo experimental.

A partir de la comparación de los resultados de pretest y postest que se muestran en la figura 4, de la información recabada en el proceso de implementación y desarrollo de la estrategia pedagógica, así como de la información sistematizada en una base de datos relacional que se construyó con el ánimo de tener una herramienta de contrastación de los datos que permitiera verificar el desarrollo y maduración del nivel de conciencia moral mostrado en el proceso de intervención pedagógica que se llevó a cabo, puede afirmarse que efectivamente se comprueban tanto la hipótesis alterna como la variable independiente planteadas en la presente investigación. Lo anterior se puede evidenciar en lo siguiente:

a) Mientras en el pretest el porcentaje alcanzado en el nivel preconvencional fue de $10,97 \%$ en el postest este porcentaje decreció a un 10,2\%, lo cual indica que hubo una disminución de $0,77 \%$ y, en tal medida, que el porcentaje restante se movió a los niveles superiores, sea convencional o posconvencional.

b) Asimismo, el porcentaje alcanzado en el pretest en el nivel convencional fue de $64,53 \%$, mientras en el postest fue de $60,83 \%$, lo que muestra una disminución de 3,7\% en este nivel. Estadísticamente, los valores en los que disminuyen estos dos primeros niveles deberían trasladarse al tercero (posconvencional). 
c) En línea con el enunciado previo, el nivel posconvencional alcanzó en el pretest un porcentaje de $24,49 \%$, que en el postest se elevó a $28,92 \%$, para un incremento de $4,43 \%$ en este nivel.

d) Es importante, asimismo, anotar que en la aplicación del postest los puntajes M y A disminuyeron significativamente. Por un lado, el M pasó de un total de 130 puntos a uno de 66 , lo que equivale a una diferencia del $49,23 \%$, y por el otro, el puntaje A pasó de 94 a un total de 75 puntos, lo que equivale a una diferencia de $20,2 \%$.

\section{Análisis general de resultados}

Se han descrito y comparado con anterioridad los resultados que se encontraron en el grupo objeto de la investigación. A continuación, trataremos de explicar, a la luz de la estrategia pedagógica desarrollada, así como de la propuesta metodológica planteada y de la teoría que fundamenta el discurso sobre el desarrollo moral, qué significan los distintos cambios en los niveles de conciencia moral mostrados por el grupo experimental en la investigación:

Por un lado, los datos reflejan unos valores en las categorías M y A menores para el momento del postest, así como un movimiento en la tendencia del grupo experimental hacia niveles superiores. Si se tiene en cuenta que los valores de las categorías $\mathrm{M}$ y A decrecieron, parece coherente afirmar, al menos metodológicamente hablando, que las respuestas dadas en el postest reflejan con mayor certeza la ubicación del grupo en los respectivos estadios de desarrollo moral que enuncia la teoría de Kohlberg. En efecto, para el análisis del postest, no hubo necesidad de eliminar ningún estudiante en virtud de su puntaje $\mathrm{M}$, pues ninguno sobrepasó la barrera de los 14 puntos porcentuales.

Diferente aconteció en el grupo de control, donde, a pesar de decrementos superiores al 50\% en las categorías $\mathrm{M}$ y A para el postest y ningún estudiante eliminado por criterio metodológico en relación al valor M>14 tanto en el pretest como en el postest, no muestran sin embargo un movimiento hacia niveles superiores de desarrollo moral tal como se supondría, lo cual se explicaría, como se expuso antes, por una respuesta dada en el pretest menos razonada que afectó el resultado final.

Al contrastar los anteriores resultados se puede afirmar que, tras las intervenciones realizadas con el grupo experimental, se dio un salto significativo en los índices de desarrollo de la conciencia moral de los estudiantes que conformaron dicho grupo. Todo esto nos lleva a plantearnos la pertinencia de la propuesta de trabajo con dilemas morales, puesto que el acercamiento a dicha estrategia pedagógica hizo evidente que favorece en los estudiantes el desarrollo de la conciencia moral.

\section{Conclusiones}

Finalmente, de acuerdo con la formulación del problema, con la hipótesis planteada y con los resultados de la investigación podemos concluir, en primer lugar, que se cumplieron los objetivos propuestos; en efecto, se estableció el nivel inicial de desarrollo de la conciencia moral en cada uno de los grupos que configuraron esta investigación a través de la aplicación del DIT de James Rest; se puso luego en práctica la estrategia pedagógica basada en dilemas morales me- 
diante las intervenciones; por último, se determinó nuevamente el nivel de desarrollo moral de los grupos experimental y de control y, en virtud de ello, se comparó dicho desarrollo en los dos momentos.

Dicha comparación hizo evidente el crecimiento en los niveles de desarrollo de la conciencia moral de los estudiantes que conformaron el grupo experimental, debido a la estrategia pedagógica (variable independiente), puesto que se presenta una disminución paralela de los niveles preconvencional y convencional, y un aumento en el posconvencional. Ello adquiere mayor significado al constatar, por ejemplo, que muchos estudiantes demostraron progresivamente un mayor nivel de argumentación frente a la mayoría de los dilemas morales planteados para cada una de las sesiones desarrolladas, tal como se puede evidenciar en la base de datos desarrollada para tal fin.

Por otro lado, aunque las investigaciones relacionadas con el desarrollo moral a partir de propuestas concretas de formación sugieren que estas tienen mayor incidencia si se aplican en periodos largos de tiempo, sería importante decir que en el caso particular de nuestra investigación, en donde el proceso de intervención tomó cerca de un año, este hecho condujo a una relativa distensión de los estudiantes del grupo experimental producida por el corte de año escolar justo en la mitad del proceso de intervención, lo cual jugó en contra del proceso, como se puede verificar por ejemplo al comparar el nivel de argumentación moral de los estudiantes durante las sesiones 4 y 5 , que se realizaron antes y después de dicho corte, respectivamente.
Las conclusiones a las que nos ha llevado este proceso de investigación y que acabamos de presentar nos permiten, a manera de corolario, ofrecer algunas recomendaciones que pueden tener relevancia en las fases posteriores del macroproyecto y en otras investigaciones que se adelanten en este ámbito:

- Resulta de vital importancia el temprano acercamiento a los diferentes postulados que tratan acerca del desarrollo de los niveles de la conciencia moral, así como la fundamentación y sugerencias metodológicas propias para el trabajo con dilemas morales. Lo anterior, dado que una mayor profundización teórica puede favorecer mejores oportunidades de aprovechamiento de las intervenciones que se vayan a realizar. Asimismo, es importante proponer un orden lógico en la aplicación de los distintos dilemas, de tal manera que se posibilite un acercamiento progresivo a situaciones morales conflictivas de mayor grado de complejidad.

- En esta misma línea, encontramos relevante determinar el periodo de tiempo en el cual se va a desarrollar la estrategia pedagógica, puesto que en los diferentes ámbitos educativos se presentan largos periodos de receso escolar que pueden incidir en el proceso que se haya adelantado previo a dichos periodos. De igual manera, es importante determinar la regularidad de las intervenciones, de tal manera que estas se realicen en intervalos similares, evitando que mientras unas se lleven a cabo con demasiada proximidad, otras se hagan en lapsos muy extensos. 


\section{REFERENCIAS BIBLIOGRÁFICAS}

Barba, B. (2001, septiembre - diciembre). Razonamiento moral de principios en estudiantes de secundaria y bachillerato. Revista Mexicana de investigación educativa.

Barba, B. (2002, 4 de julio). Influencia de la edad y la escolaridad en el desarrollo del juicio moral. Revista electrónica de investigación educativa. Universidad Autónoma de Aguas Calientes.

Basanta, E. M. (s.f.). La formación ética en las instituciones de educación superior. Argentina: Universidad Nacional de La Matanza. Versión en línea: www.rieoei. org/deloslectores/173Basanta.PDF.

Becattini, M. (1999). La formazione morale nel fanciullo. Tesi di Laurea. Urbino: Università di Urbino, Facoltà di Scienze dell'Educazione. Versión en línea: http://www.tesionline.it/default/tesi. asp?idt=12250.

Bermúdez, Á. y Jaramillo, R. (2000). El análisis de dilemas morales. Una estrategia pedagógica para el desarrollo de la autonomía moral. Bogotá: Corpoeducacion.

Buendía, L. (1998). Métodos de investigación en psicopedagogía. Madrid: Mc. Graw Hill.

Castorina, L. (2007). Lo sviluppo del giudizio morale. Ricerca su un campione siciliano e confronto dei dati con un campione del nord Italia. Tesi di Laurea. Padua: Università di Padova, Facoltà di Psicologia. Versión en línea: http://www.tesionline.it/default/tesi. asp?idt=18661.

Coppens, M. (2008). Les dilemmes moraux dans le cours de morale. Diotime, Revue Internationale de Didactique de la Philoso- phie. Academie de Montpellier. Versión en línea: http://www.crdp-montpellier.fr/ ressources/agora/D016053A.HTM.

Dal Canton, I. (2002). Conflitto e comunicazione. Per una critica di Jürgen Habermas. Milán: Edizioni Ghibli. Versión en línea: http://books.google.com.co.

Daukins, R. (1993). El gen egoísta. Las bases biológicas de nuestra conducta. Barcelona: Salvat.

De Bennato, A. (2009). "La intervención ético-pedagógica en la formación docente. Una propuesta para su tematización y aplicación" Instituto de Filosofía, Universidad Nacional del Nordeste - Argentina. Versión en línea: www.unne. edu.ar/cyt/2001/9-Educacion/D-018. pdf.

De Waal, F. (2007). Primates y filósofos. La evolución de la moral del simio al hombre. Barcelona: Paidós.

Dreyfus, H. L. (1998). What is Moral Maturity? A Phenomenological Account of the Development of Ethical Expertise. Berkeley, University of California. Versión en línea: http://www.nuc.berkeley.edu/ courses/classes/E-124/Moral_Maturity_8_90.pdf.

Gibbs, J.C. \& Schnell, S.V. (1985). Moral development versus socialization: A critique. American Psychologist, 40, 10711080. Citado en Wenek, K.W.J. (1996). Une perspective psychologique du développement moral.

González, L. J. (2003). Ética. Bogotá: El Búho.

Hauser, M. (2006). Moral Minds. How nature designed our universal senses of right and wrong? New York: Ecco. Citado por: Musco, S. (2008). Buoni per natura. Le ra- 
HALLAZGOS - Revista de Investigaciones • Año 7 - No. 13 • Enero-junio de 2010

dici evolutive della coscienza morale. Tesi di Laurea. Catanzaro, Università della Calabria, Facoltà de Lettere e Filosofia. Versión en línea: http://www.tesionline. it/default/tesi.asp?idt=27015.

Hernández P., F. (1998). Diseños de investigación experimental. Métodos de investigación en Psicopedagogía. Madrid: Mc. Graw Hill.

Hersh, R., Reimer, J. \& Paolitto, D. (1998). El crecimiento moral. De Piaget a Kohlberg. Madrid: Narcea.

Kohlberg, L. (1983). La comunidad justa en el desarrollo moral. Educación hoy, 7578, 20 ss.

Kolakowski, L. (1993, 19 de abril). Utopía y futuro. El País.

Lehalle, H., Aris, C., Buelga, S., \& Musitu, G. (2004). Développement socio-cognitif et jugement moral. L'orientation scolaire et professionnel, 33, 289-314. Versión en línea: http://osp.revues.org/index2163. html.

López A., S. (2008, mayo). Anejo: Auge y caída de la teoría de la capa. El Viejo Topo, 244. Barcelona.

Musco, S. (2008). Buoni per natura. Le radici evolutive della coscienza morale. Catanzaro. Tesi di Laurea. Università della Calabria, Facoltà de Lettere e Filosofia. Versión en línea: http://www.tesionline. it/default/tesi.asp?idt=27015.

Oser, F. (1998). El hombre: estadios del desarrollo religioso. Una aproximación desde el estructuralismo genético. Barcelona: Ariel.

Oser, F. (2005). Futuras perspectivas de la educación moral. Organización de Estados Iberoamericanos. Versión en línea: http://www.campus-oei.org/oeivirt/rie08a01.htm.
Ouellet, F. (Director). (2006). Quelle formation pour l'enseignement de l'éthique à l'école? Québec: Presses de 1'Université Laval. Versión en línea: http://books. google.com.co.

Pagoni-Andréani, M. (1999). Le dévelopment socio-moral: des Théories à l'éducation civique. Villeneuve-d'Aseq (Nord): Presses Universitaires du Septentrion. Versión en línea: http://books.google. com.co.

Pompa, A., Pérez, G., López, E., Nicolás P., B., Mohar, F., Rouco, B., Fernández, N., Mendibur, F. \& Trujillo, A. (2001). “Formación de valores en los estudiantes universitarios cubanos. Antecedentes y visión de un proyecto". Versión en línea: http://www.umcc.cu/pu/pedagogia\%202001.htm.

Riechmann, J. (2009). (en prensa). ¿Somos los seres humanos egoístas por naturaleza?. La habitación de Pascal. Ensayos para fundamentar éticas de suficiencia $y$ politicas de autocontención. Madrid: Los libros de la Catarata. Versión en línea: http://www.scribd.com/doc/17280913/ EGOISMO.

Schlaefli, A., Rest, J. R. \& Thoma, S. J. (1985). Does moral education improve moral judgment? A meta-analysis of intervention studies using the Defining Issues Test. Review of Educational Research, 55, 319-52. Último acceso: 18/01/2010, a través de sistema de información ProQuest.

Suárez, G. \& Meza, J. L. (2005). Conciencia moral. Estudio del desarrollo de la conciencia moral y propuesta para su formación en la educación superior. Informe final de investigación. Grupo Ambiente ético. Bogotá: Universidad de La Salle. 
Suarez, G. \& Meza, J. (2008). Efecto de una estrategia pedagógica en el desarrollo de la conciencia moral en un grupo de estudiantes de educación superior. Maestría en Docencia. Bogotá: Universidad de La Salle.

Thoma, S., \& Rest, J. (1999). The relationship between moral decision making and patterns of consolidation and transition in moral judgment development. Developmental Psychology, 35, №. 2, 323-334.

Vargas, G. (2004). La representación computacional de dilemas morales. Investigación fenomenológica de epistemología experimental. Bogotá: Universidad Pedagógica Nacional.
Villegas, C. (1998). Influencia de Piaget en el estudio del desarrollo moral. Revista latinoamericana de psicología, 30, No. 2, 223-232.

Wenek, K. (1996, 24 y 25 de octubre). Une perspective psychologique du développement moral. Les multiples faces éthiques de la défense. Actes de la Conférence sur l'Éthique dans la défense canadienne, 83-97. Ottawa: Programme d'Éthique de la Défense, Quartier Général de la Défense Nationale. Versión en línea: http://www.dep-ped.forces.gc.ca/ publications/documents/conf1996-fra. pdf\#page $=90$

Wren, T.E. (Editor). (1990). The moral domain. Essays in the ongoing discussion between Phylosophy and Social Sciences. Massachusetts: Massachusetts Institute of Technology. Versión en línea: http:// books.google.com.co. 